\title{
Bone marrow-derived mesenchymal stem cells protect against lung injury in a mouse model of bronchopulmonary dysplasia
}

\author{
YUN LUAN ${ }^{1}$, WEI DING ${ }^{2}$, ZHI-YE JU $^{2}$, ZHAO-HUA ZHANG $^{3}$, XUE ZHANG $^{3}$ and FENG KONG ${ }^{1}$ \\ ${ }^{1}$ Central Research Laboratory, The Second Hospital of Shandong University, Jinan, Shandong 250000; \\ ${ }^{2}$ Department of Radiology, Rizhao City People's Hospital, Rizhao, Shandong 276807; \\ ${ }^{3}$ Department of Pediatrics, The Second Hospital of Shandong University, Jinan, Shandong 250000, P.R. China
}

Received February 14, 2014; Accepted November 4, 2014

DOI: $10.3892 / \mathrm{mmr} .2014 .2959$

\begin{abstract}
The aim of the present study was to investigate the effect of bone marrow-derived mesenchymal stem cells (BMSCs) in the treatment of lung injury in a mouse model of bronchopulmonary dysplasia (BPD) and examine the underlying mechanisms. A mouse model of BPD was created using continuous exposure to high oxygen levels for 14 days. BMSCs were isolated, cultured and then labeled with green fluorescent protein. Cells $\left(1 \times 10^{6}\right)$ were subsequently injected intravenously $1 \mathrm{~h}$ prior to high oxygen treatment. Animals were randomly divided into three groups $(\mathrm{n}=5$ in each): Control group, BPD model group and BMSC injection group. At two weeks post-treatment, the expression of transforming growth factor- $\beta 1$ (TGF- $\beta 1$ ), vascular endothelial growth factor (VEGF) and von Willebrand factor (vWF) was detected using immunohistochemical staining and immunofluorescence. Compared with the BPD model group, the body weight, airway structure and levels of TGF- $\beta 1$ and VEGF were significantly improved in the BMSC-treated group. Immunofluorescence observations indicated that BMSCs were able to differentiate into cells expressing vWF and VEGF, which are markers of vascular tissues. The present study demonstrated that intravenous injection of BMSCs significantly improved lung damage in a neonatal mouse model of BPD at 14 days following hyperoxia-induced injury. This provides novel information which may be used to guide further investigation into the use of stem cells in BPD.
\end{abstract}

\section{Introduction}

Bronchopulmonary dysplasia (BPD) is the most common form of chronic lung disorder in babies born prematurely and

Correspondence to: Dr Feng Kong, Central Research Laboratory, The Second Hospital of Shandong University, 247 Beiyuan Dajie, Jinan, Shandong 250000, P.R. China

E-mail: fengk1970@163.com

Key words: bone marrow-derived mesenchymal stem cells, bronchopulmonary dysplasia, lung causes ongoing morbidity and mortality, characterized by impaired alveolar development and inflammation (1). There is currently no effective intervention with which to prevent or treat bronchopulmonary dysplasia (2). Stem cell therapy is a potential therapeutic approach for BPD and studies have shown that BMSCs protect the lung and aid in the repair of injured lung tissues in a number of animal models $(3,4)$. Studies have also demonstrated that intravenous or intratracheal administration of BMSCs protects against hyperoxic lung injury by reducing inflammation and improving alveolar structure $(5,6)$. To date, the mechanisms underlying these effects remain elusive. It is likely that paracrine-mediated mechanisms and immunomodulation (7) are involved, amongst other unidentified processes.

Transforming growth factor- $\beta$ (TGF- $\beta$ ) signaling and the pathways in which it is involved are known to be important during lung development (8). Increased expression of TGF- $\beta$ inhibits development of the alveoli and also causes pulmonary fibrosis in lungs with advanced alveolarization. Overexpression of TGF- $\beta$ leads to structural changes associated with BPD, including the proliferation of $\alpha$-actin-positive myofibroblasts within the alveolar septal walls, the development of abnormal alveolar structure and vascular development in the neonatal mouse lung $(9,10)$. Vascular endothelial growth factor (VEGF) is also known to be involved in the regulation of pulmonary vascular growth and development, the stimulation of angiogenesis and the promotion of endothelial survival (11). Inhibition of VEGF receptor-2 (VEGFR2) in neonatal rats causes rarefaction of pulmonary vessels and impairs alveolar formation (12), whereas enhancement of VEGF signaling ameliorates the alveolar disruption induced by hyperemia (13). The present study investigated the effect of BMSCs in the treatment of lung injury in a mouse model of BPD and examined whether the underlying mechanism was via the abovementioned signaling pathways.

\section{Materials and methods}

Animal. Neonatal C57BL/6 mice (24 h old; weight, 1-2 g; $\mathrm{n}=15$ ), used in all experiments, were obtained from the Animal Experiment Center of Shandong University (Shandong, China). The animals were kept in a temperature-controlled environment $\left(22-24^{\circ} \mathrm{C}\right)$ under a 12 -h light/dark cycle with access to food and water ad libitum. Mice were randomized into various 
groups. They were then weighed and blood samples were collected. All animal procedures were approved by the animal ethics committee of Shandong University (Jinan, China) and followed the Guide for the Care and Use of Laboratory Animals published by the US National Institutes of Health (NIH Publication no. 85-23, revised 1996).

Culture, immunophenotype analysis and lentiviral transfection with green fluorescent protein (GFP) of BMSCs. BMSCs were isolated from the femur of six to eight-week-old C57BL/6 mice, as previously described (14). Briefly, fresh bone marrow cells were isolated by flushing Dulbecco's modified Eagle's medium (DMEM; Gibco-BRL, Carlsbad, CA, USA) containing $1 \%$ penicillin-streptomycin (Sigma-Aldrich, St Louis, MO, USA) through the medullary cavity of mouse femurs. Cells were isolated using the Ficoll (1.077; Sigma-Aldrich) density gradient centrifugation method. The isolated bone marrow cells were then cultured and expanded in a low-glucose culture containing DMEM with F-12 (LG-DMEM; Gibco-BRL, Life Technologies, Carlsbad, CA, USA), supplemented with $10 \%$ fetal bovine serum (Hyclone, Logan, UT, USA), $100 \mathrm{U} / \mathrm{ml}$ penicillin and $100 \mathrm{~g} / \mathrm{ml}$ streptomycin (Sigma-Aldrich). All cultures were maintained at $37^{\circ} \mathrm{C}$ in $5 \%$ humidified $\mathrm{CO}_{2}$. Characteristic immunoreactivity for cell markers was detected using fluorescent activated cell sorting analysis (FACS; FACScan BD LSRFortessa; BD Biosciences, Franklin Lakes, NJ, USA) with rat monoclonal anti-mouse anitbodies for CD44, CD117, CD34 and CD106 (Santa Cruz Biotechnology, Inc., Dallas, TX, USA). Briefly, cells growing on a poly-L-lysine-coated 24-well plate (Sigma-Aldrich) were washed three times with phosphate-buffered saline (PBS; Sigma-Aldrich) and fixed in 4\% paraformaldehyde for $30 \mathrm{~min}$. Cells were then permeabilized with $0.1 \%$ Triton X-100 in PBS for 20 min and blocked with $4 \%$ goat serum for $1 \mathrm{~h}$. Cells were incubated with fluorescein isothiocyanate-conjugated goat-anti-rat secondary antibodies for $1 \mathrm{~h}$ at room temperature in the absence of sunlight.

GFP was cloned into a lentiviral vector using the Ubiquitin C promoter, as described previously (15). In brief, virus particles were packaged in 293FT cells (Shanghai Genechem Co., Ltd., Shanghai, China) in vitro and condensed by ultracentrifugation ( 600 x g, $5 \mathrm{~min}$ ). Green fluorescence was observed after $48 \mathrm{~h}$ using a fluorescence microscope (Nikon Elcipse 90i; Nikon Corp., Tokyo, Japan), and the medium was replaced every two days with fresh medium. The cells were transferred when they reached $80 \%$ confluence.

Preparation of a mouse model of BPD mice. The mice were prepared as previously described (16). Briefly, C57BL/6 mice $(n=5)$ were placed in chambers, in which the oxygen concentration was maintained at $\mathrm{FiO}_{2}=0.60$. Exposure to hyperoxia was continuous, and mice were maintained in a hypoxic environment for $14 \mathrm{~d}$, with a brief interruption for animal care (less than $10 \mathrm{~min} /$ day). BMSCs $\left(1 \times 10^{6}\right)$ were administered intravenously at $1 \mathrm{~h}$ prior to establishment of the BPD model.

Lung histology and morphometric analysis. Lungs were prepared for histological examination as previously described (17). Briefly, mice were sacrificed by intraperitoneal injection of pentobarbital (100 mg/kg; Sigma-Aldrich) when they were 14 days old. Lungs were fixed with $4 \%$ paraformaldehyde solution overnight, and the left lower lobe was embedded in paraffin. Sections were cut with a microtome set at 4-5 $\mu \mathrm{m}$ (Leica RM226, Leica Microsystems, Wetzlar, Germany). Alveolarization was assessed by performing radial alveolar counts (RAC) according to standard methods as previously described (17). Briefly, from the center of the respiratory bronchiole a perpendicular line was drawn to the edge of the acinus (as defined by a connective tissue septum or the pleura). The number of septa intersected by this line was counted. Five counts were performed for each animal; the average of the five high-power fields (hpf) was randomly selected. These experiments were conducted by two examiners, blinded to treatment assignment.

Immunohistochemical staining. At 14 days following the operation, mice were anesthetized, lungs were fixed in $4 \%$ paraformaldehyde/PBS for $24 \mathrm{~h}$ and then stored in $70 \%$ ethanol. The left lower lobe was embedded in paraffin, cut into sections of 4-5 $\mu \mathrm{m}$, deparaffinized in xylene and then rehydrated by serial immersions in $100 \%$ ethanol, $95 \%$ ethanol, $85 \%$ ethanol, $75 \%$ ethanol (Sigma-Aldrich) and $100 \%$ water. Slides were blocked with $4 \%$ normal goat serum in PBS, then incubated with primary antibodies overnight at $4^{\circ} \mathrm{C}$ and a biotinylated secondary antibody for $20 \mathrm{~min}$. Immunohistochemistry and immunofluorescence were conducted with rabbit monoclonal anti-mouse TGF- $\beta 1$ (1:100; Santa Cruz Biotechnology, Inc.), VEGF (1:150; Abcam, Cambridge, MA, USA) and vWF (1:150; Abcam) immunoglobulin G. Fluorescein isothiocyanate-conjugated goat anti-rabbit secondary antibodies were then used (1:200, Abcam, Cambridge, UK). Staining was quantified using the image-analysis system Image Pro Plus 6.0 (Media Cybernetics, Rockville, MD, USA) as previously described (18).

Quantitative reverse transcription-polymerase chain reaction ( $q R T-P C R)$ analysis. Once the lungs had been harvested, total RNA was extracted from lung tissue homogenates (Invitrogen Life Technologies). qRT-PCR analysis was conducted as previously described (18) in order to detect the relative pulmonary expression levels of TGF- $\beta 1$ and VEGF. RNA samples were dissolved in RNase-free water and quantified spectrophotometrically (BiochromDNA UV-Vis, WPA; Biochrom,Ltd.,Cambridge, UK).Primers were designed using the Primer Express software package (Applied Biosystems, Foster City, CA, USA). The following primers were used: Forward, 5'-AGGTAACGCCAGGAATGTTGCTA-3' and reverse, 5'-CATTGCTGTCCCGTGCAGA-3' for TGF- $\beta 1$; forward, 5'-CTGCTCTCCTGGGTGCATTG-3' and reverse, 5'-ACTCCTGGAAGATGTCCACCA-3' for VEGF; and forward, 5'-ACTCTGGCAAAGTGGATATTGTCG-3' and reverse, 5'-CAGCATCACCCCATTTGATG-3' for GAPDH. Data were analyzed using the ABI Prism 7900 sequence detection system software (version 2.2; Applied Biosystems).

Statistical analysis. All data are expressed as the mean \pm standard error of the mean. Comparisons of parameters between two groups were made using unpaired Student's $t$-test. Statistical analysis was conducted using the SPSS 13.0 software (SPSS, Inc., Chicago, IL, USA). P $<0.05$ was considered to indicate a statistically significant difference. 


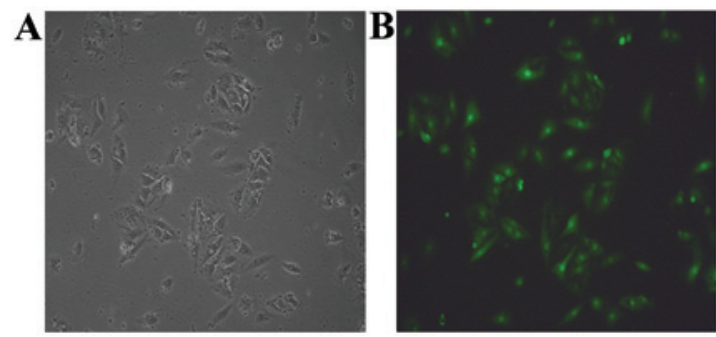

Figure 1. Bone marrow-derived mesenchymal stem cells from C57BL/6 mice at day 2 following transfection with GFP. (A) Normal and (B) GFP. Magnification, x200. GFP, green fluorescent protein.

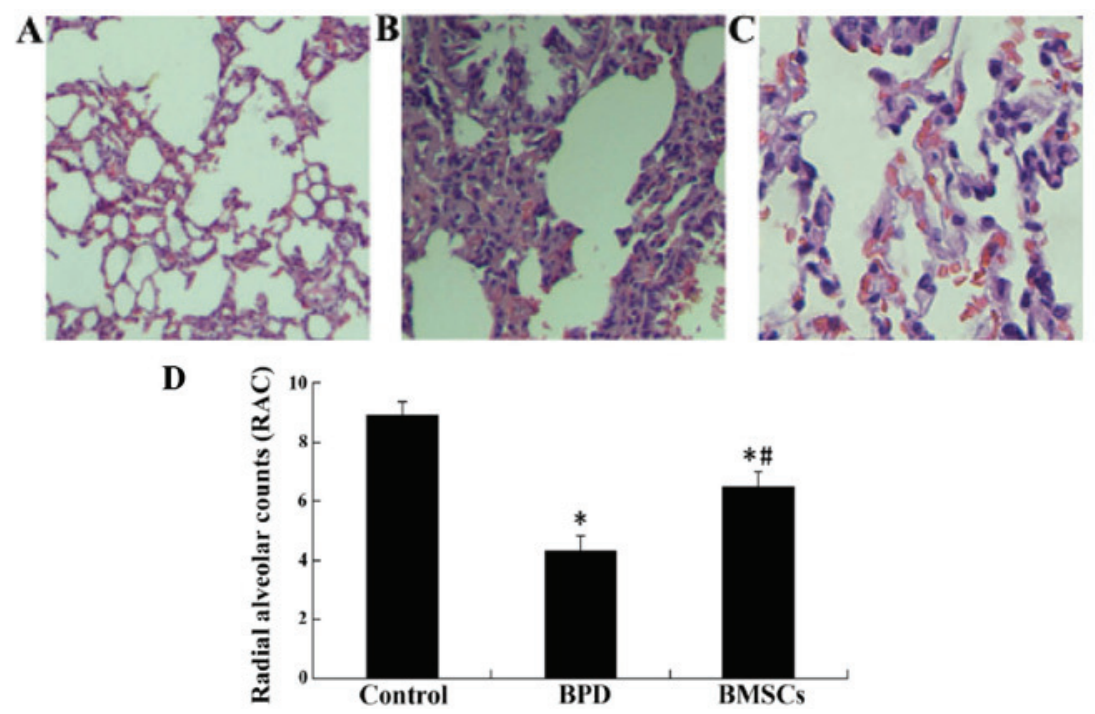

Figure 2. Hematoxylin and eosin staining of lung tissue. Effects of BMSC treatment on lung injury in a mouse model of BPD at 14 days of age. (A) Control group, (B) BPD group and (C) BMSC group. (D) RAC for each group. Data are presented as the mean \pm standard error of the mean ( $\mathrm{n}=5$ ). " $\mathrm{P}<0.05$ compared with control group and " $\mathrm{P}<0.05$ compared with BPD group. Magnification, $\mathrm{x} 200$. BMSCs, bone marrow-derived mesenchymal stem cells; BPD, bronchopulmonary dysplasia; RAC, radial alveolar counts.

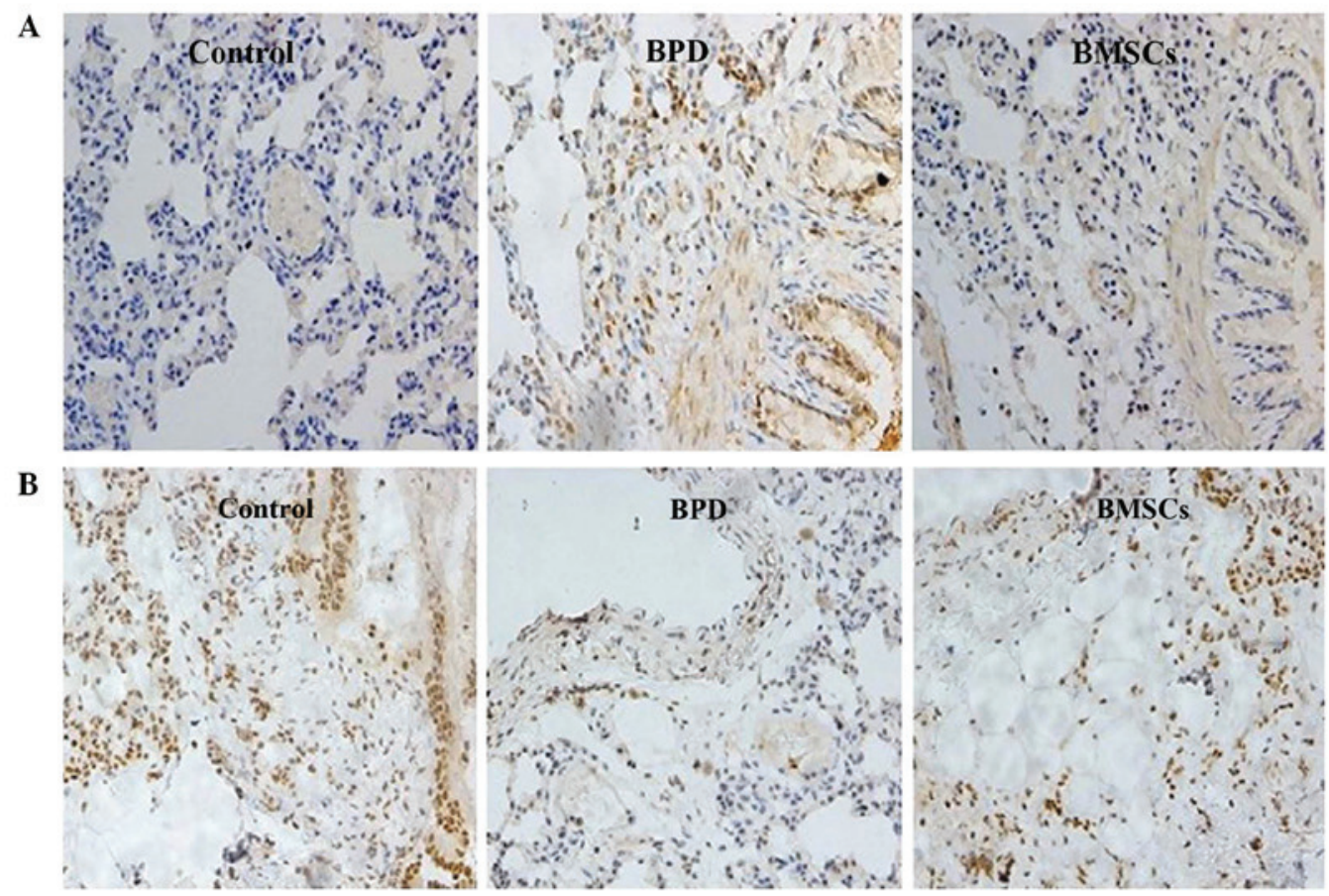

Figure 3. Immunohistochemical staining: Protein expression of (A) transforming growth factor- $\beta 1$ and (B) vascular endothelial growth factor in lung tissue. Positive staining of cytoplasm is yellow-brown and that of nuclei is blue. Magnification, x200. BPD, bronchopulmonary dysplasia; BMSCs, bone marrow-derived mesenchymal stem cells. 
Table I. Body weight in each group $(n=5)$.

\begin{tabular}{lcr}
\hline & & Weight $(\mathrm{g})$ \\
\cline { 2 - 3 } Group & Day 0 & Day 14 \\
\hline Control & $1.93 \pm 0.19$ & $10.96 \pm 0.23$ \\
BPD & $1.92 \pm 0.16$ & $8.73 \pm 0.27^{\mathrm{a}}$ \\
BMSCs & $1.92 \pm 0.20$ & $10.96 \pm 0.21^{\mathrm{a}, \mathrm{b}}$ \\
P-value & $>0.05$ & $<0.05$ \\
\hline
\end{tabular}

Data are presented as the mean \pm standard error of the mean $(n=5) .{ }^{a} \mathrm{P}<0.05$ compared with control group; ${ }^{\text {b }}<<0.05$ compared with $\mathrm{BPD}$ group BPD, bronchopulmonary dysplasia; BMSCs, bone marrow-derived stem cells.

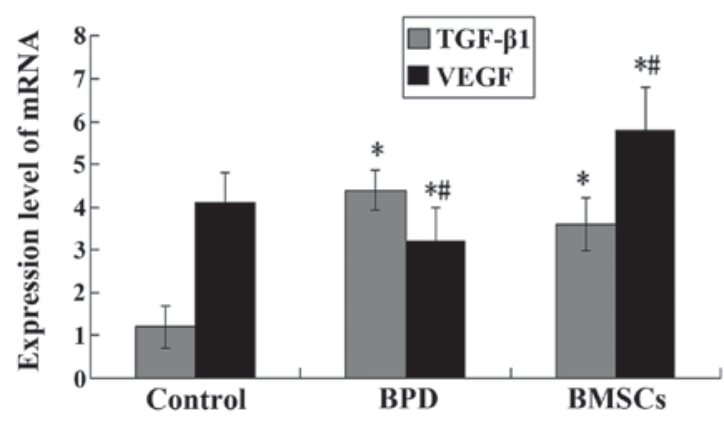

Figure 4. Quantitative reverse transcription polymerase chain reaction analysis of mRNA levels of TGF- $\beta 1$ and VEGF in lung tissue. Data are presented as the mean \pm standard error of the mean $(n=5)$. ${ }^{*} \mathrm{P}<0.05$, compared with the control group and ${ }^{\#} \mathrm{P}<0.05$, compared with the hyperoxia group. TGF- $\beta 1$, transforming growth factor- $\beta 1$; VEGF, vascular endothelial growth factor.

\section{Results}

Characterization of cultured BMSCs and transfection with GFP. Following primary culture, BMSCs appeared as spindle-like cells attached to the tissue culture dishes. Three days after being subcultured, cells were observed to be tightly adherent to the culture dish and proliferated rapidly in the culture medium. GFP-transfected cells from C57BL/6 mice expressed green fluorescence at day 2 (Fig. 1). The surface markers of BMSCs, determined by FAC, showed that BMSCs were positive for CD44 and CD106, but negative for CD34 and CD117.

Body weight. Each time-point in each study group was represented by five animals. The average body weight was lower in the BPD group at 14 days of age $(8.73 \pm 0.27 \mathrm{~g})$ compared with that of the control group at the same age $(10.96 \pm 0.21 \mathrm{~g} ; \mathrm{P}<0.05)$. The group treated with BMSCs exhibited a higher average body weight $(9.18 \pm 0.26 \mathrm{~g})$ in comparison with that in the BPD group $(\mathrm{P}<0.05$; Table I).

Lung histology and morphometrics. In order to investigate whether BMSCs produce beneficial effects on hyperoxia-induced impairments in lung structure, a morphometric analysis was conducted using RAC. The RAC were significantly lower in the BPD group than they were in the control group. However, they were significantly higher in the group treated with BMSCs than those in the BPD group, 14 days of age $(\mathrm{P}<0.05$; Fig. 2$)$.
TGF- $\beta 1$ and VEGF protein expression in lung tissue. Immunohistochemical staining analysis demonstrated that exposure of neonatal mice to hyperoxia resulted in a marked increase in expression of the TGF- $\beta 1$ protein in the lung, but a decrease in VEGF expression at 14 days of age. These changes in protein levels proteins were visibly abrogated at day 14 following treatment with BMSCs (Fig. 3).

TGF- $\beta 1$ and VEGF mRNA expression in lung tissue. The results from the qRT-PCR analysis showed that the levels of VEGF mRNA in the lungs were significantly lower, and those of TGF- $\beta 1$ mRNA were significantly higher in the BPD group compared with those in the control group at 14 days of age. TGF- $\beta 1$ levels were significantly decreased in the group treated with BMSCs compared with those in the BPD group ( $\mathrm{P}<0.05$; Fig. 4$)$.

Identification of the injected BMSCs. At 14 days following injection of the cells into C57BL/6 mice, immunocytochemistry staining for vessel markers, vWF (red) and VEGF (red), in lung tissue was conducted. The images were captured with a camera system connected to a fluorescence microscope. The results indicated that the injected BMSCs were able differentiate into vascular endothelial cells in vivo (Fig. 5).

\section{Discussion}

The results of the present study showed that intravenous implantation of BMSCs $\left(\times 10^{6}\right)$ improved the lung impairment 

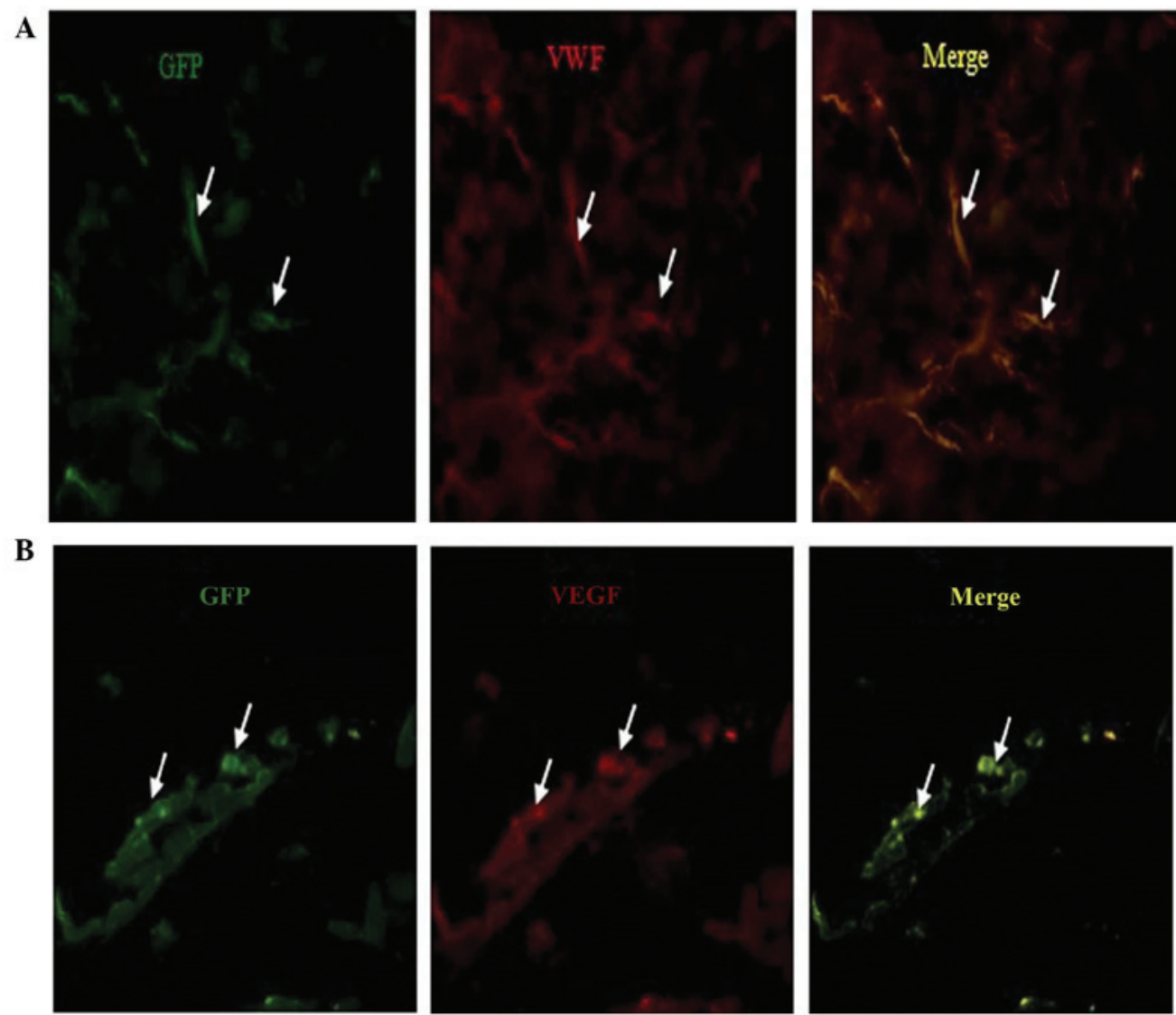

Figure 5. Identification of the intravenous injection of BMSCs by fluorescence microscopy. At 14 days of age, the GFP-labeled cells were identified by observing the distribution of GFP-positive cells. Sections stained for (A) vWF and (B) VEGF exhibited red fluorescence, and in the merged images, staining is displayed in yellow, with white arrows indicating BMSCs expressing vWF and VEGF. Magnification, x400. BMSCs, bone marrow-derived mesenchymal stem cells; GFP, green fluorescent protein; vWF, von Willebrand factor; VEGF, vascular endothelial growth factor.

caused by exposure to hyperoxia. Treatment with BMSCs in a mouse model of BPD significantly increased the average body weight and improved the airway structure in hyperoxia-damaged lungs. Administration of BMSCs also resulted in a reduced expression of TGF- $\beta 1$ and an increased expression of VEGF compared with those in the BPD group. Immunofluorescence staining indicated that intravenously implanted BMSCs were capable of differentiating into cells expressing vWF and VEGF, which are markers of vascular tissues, at 2 weeks in vivo post-injection. These results may provide novel therapeutic avenues for lung diseases that are currently lacking efficient treatments.

Intravenous or intra-alveolar administration of BMSCs attenuates the severity of lung damage following bleomycin and endotoxin-induced lung injury in adult rats (19,20). Recent studies have indicated that BMSCs may prevent arrested alveolar and vascular growth, in part through paracrine activity, in experimental neonatal lung injury models in rats (5). Potential mechanisms by which treatment with BMSCs improves lung structure include engraftment, anti-inflammatory and immunomodulatory functions, and antiapoptotic effects. However, there are a number of questions remaining (21), which require further investigation, and little is currently known regarding the potential role of BMSCs in the setting of neonatal lung injury (22). BMSCs migrate to, or participate in the development of, lung tissue (23-25). A number of studies have demonstrated that stem/progenitor cells have the potential to be used as cellular therapies to contribute to lung repair mechanisms following acute lung injury.

TGF- $\beta$ and its downstream mediators have been implicated in the development of $\operatorname{BPD}(26,27)$, and TGF- $\beta$ signaling is important in normal lung development and the lung injury/repair response. TGF- $\beta$ signaling is initiated by ligand-induced serine/threonine receptor kinases and phosphorylation of the cytoplasmic signaling molecules, the Smad family. BPD may result from abnormal differentiation of alveolar mesenchymal progenitor cells into myofibroblasts under the influence of TGF- $\beta$. The findings of the present study support the hypothesis that TGF- $\beta$ may be involved in BPD. VEGF is also known to be important in the regulation of pulmonary vascular growth and development, and for stimulating angiogenesis and promoting endothelial survival (28). The expression levels of VEGF were reduced in animal models of BDP and in the lungs of premature infants, in whom BPD was the cause of mortality (29-31). Inhibition of VEGF receptor-2 (VEGFR2) has been shown to cause rarefaction of pulmonary vessels and impairment in alveolar formation (32) in neonatal rats, whereas enhancement of VEGF signaling ameliorated the alveolar disruption induced by hyperemia (33). Furthermore, blocking postnatal angiogenesis impaired alveolarization, and decreased pulmonary capillary density was observed in animal models and patients with BPD (34). 
The results of the present study are in accordance with these previous studies. Expression levels of TGF- $\beta 1$ protein and mRNA were significantly decreased, and those of VEGF were significantly increased, at 14 days after intravenous injection of BMSCs. Furthermore, the results of the immunofluorescence experiments showed that BMSCs were able to differentiate into cells expressing vWF and VEGF at day 14. These results suggested that intravenously implanted BMSCs had the ability to differentiate into vascular endothelial cells in vivo. Therefore, the protective mechanisms underlying the effects of treatment with BMSCs in a mouse model of BPD included homing of these cells to the lung, where they differentiated into vascular endothelial cells, or through paracrine effects.

In conclusion, the present study demonstrated that intravenous injection of BMSCs significantly improved the damaged airway structure and the levels of TGF- $\beta 1$ and VEGF in the lungs of mice with BPD.

\section{Acknowledgements}

This study was supported by a grant from the Youth Foundation of the Second Hospital of Shandong University (grant no. Y2013010068).

\section{References}

1. Rezvani M, Wilde J, Vitt P, et al: Association of a FGFR-4 gene polymorphism with bronchopulmonary dysplasia and neonatal respiratory distress. Dis Markers 35: 633-640, 2013.

2. Sakurai R, Li Y, Torday JS and Rehan VK: Curcumin augments lung maturation, preventing neonatal lung injury by inhibiting TGF- $\beta$ signaling. Am J Physiol Lung Cell Mol Physiol 301: L721-L730, 2011

3. Xu J, Woods CR, Mora AL, et al: Prevention of endotoxin-induced systemic response by bone marrow-derived mesenchymal stem cells in mice. Am J Physiol Lung Cell Mol Physiol 293: L131-L141, 2007.

4. Ortiz LA, Gambelli F, McBride C, et al: Mesenchymal stem cell engraftment in lung is enhanced in response to bleomycin exposure and ameliorates its fibrotic effects. Proc Natl Acad Sci USA 100: 8407-8411, 2003.

5. van Haaften T, Byrne R, Bonnet S, et al: Airway delivery of mesenchymal stem cells prevents arrested alveolar growth in neonatal lung injury in rats. Am J Respir Crit Care Med 180: 1131-1142, 2009.

6. Aslam M, Baveja R, Liang OD, et al: Bone marrow stromal cells attenuate lung injury in a murine model of neonatal chronic lung disease. Am J Respir Crit Care Med 180: 1122-1130, 2009.

7. Lee JW, Fang X, Krasnodembskaya A, Howard JP and Matthay MA: Concise review: Mesenchymal stem cells for acute lung injury: role of paracrine soluble factors. Stem Cells 29: 913-919, 2011.

8. Gauldie J, Galt T, Bonniaud P, et al: Transfer of the active form of transforming growth factor-beta 1 gene to newborn rat lung induces changes consistent with bronchopulmonary dysplasia. Am J Pathol 163: 2575-2584, 2003.

9. Vicencio AG, Lee CG, Cho SJ, et al: Conditional overexpression of bioactive transforming growth factor-beta 1 in neonatal mouse lung: a new model for bronchopulmonary dysplasia? Am J Respir Cell Mol Biol 31: 650-656, 2004.

10. Monz D, Tutdibi E, Mildau C, et al: Human umbilical cord blood mononuclear cells in a double-hit model of bronchopulmonary dysplasia in neonatal mice. PLoS One 8: e74740, 2013.

11. Abman SH: Impaired vascular endothelial growth factor signaling in the pathogenesis of neonatal pulmonary vascular disease. Adv Exp Med Biol 661: 323-335, 2010.

12. Jakkula M, Le Cras TD, Gebb S, et al: Inhibition of angiogenesis decreases alveolarization in the developing rat lung. Am J Physiol Lung Cell Mol Physiol 279: L600-L607, 2000.
13. Acarregui MJ, Penisten ST, Goss KL, Ramirez K and Snyder JM: Vascular endothelial growth factor gene expression in human fetal lung in vitro. Am J Respir Cell Mol Biol 20: 14-23, 1999.

14. Okabe M, Ikawa M, Kominami K, et al: 'Green mice' as a source of ubiquitous green cells. FEBS Lett 407: 313-319, 1997.

15. Peng C, Yang K, Xiang P, et al: Effect of transplantation with autologous bone marrow stem cells on acute myocardial infarction. Int J Cardiol 162: 158-165, 2013.

16. Balasubramaniam V, Mervis CF, Maxey AM, Markham NE and Abman SH: Hyperoxia reduces bone marrow, circulating and lung endothelial progenitor cells in the developing lung: implications for the pathogenesis of bronchopulmonary dysplasia. Am J Physiol Lung Cell Mol Physiol 292: L1073-L1084, 2007.

17. Kunig AM, Balasubramaniam V, Markham NE, Seedorf G, Gien J and Abman SH: Recombinant human VEGF treatment transiently increases lung edema but enhances lung structure after neonatal hyperoxia. Am J Physiol Lung Cell Mol Physiol 291: L1068-L1078, 2006.

18. Luan Y, Zhang ZH, Wei DE, Lu Y and Wang YB: Effects of autologous bone marrow mononuclear cells implantation in canine model of pulmonary hypertension. Circ J 76: 977-985, 2012.

19. Rojas M, Xu J, Woods CR, et al: Bone marrow derived mesenchymal stem cells in repair of the injured lung. Am J Respir Cell Mol Biol 33: 145-152, 2005

20. Ortiz LA, Gambelli F, McBride C, et al: MSC engraftment in lung is enhanced in response to bleomycin exposure and ameliorates its fibroproliferative effects. Proc Natl Acad Sci USA 100: 8407-8011,2003.

21. Abman SH and Matthay MA: Mesenchymal stem cells for the prevention of bronchopulmonary dysplasia: delivering the secretome. Am J Respir Crit Care Med 180: 1039-1041, 2009.

22. Hennrick KT, Keeton AG, Nanua S, et al: Lung cells from neonates show a mesenchymal stem cell phenotype. Am J Respir Crit Care Med 175: 1158-1164, 2007

23. Jiang Y, Jahagirdar BN, Reinhardt RL, et al: Pluripotency of mesenchymal stem cells derived from adult marrow. Nature 418: 41-49, 2002.

24. Krause DS, Theise ND, Collector MI, et al: Multi-organ, multi-lineage engraftment by a single bone marrow-derived stem cell. Cell 105: 369-377, 2001.

25. Wen ST, Chen W, Chen HL, et al: Amniotic fluid stem cells from EGFP transgenic mice attenuate hyperoxia-induced acute lung injury. PLoS One 8: e75383, 2013.

26. Collins JJ, Kallapur SG, Knox CL, et al: Repeated intrauterine exposures to inflammatory stimuli attenuated transforming growth factor- $\beta$ signaling in the ovine fetal lung. Neonatology 104 : 49-55, 2013.

27. Collins JJ, Kunzmann S, Kuypers E, et al: Antenatal glucocorticoids counteract LPS changes in TGF- $\beta$ pathway and caveolin-1 in ovine fetal lung. Am J Physiol Lung Cell Mol Physiol 304: L438-L444, 2013.

28. Abman SH: Impaired vascular endothelial growth factor signaling in the pathogenesis of neonatal pulmonary vascular disease. Adv Exp Med Biol 661: 323-335, 2010.

29. De Paepe ME, Mao Q, Powell J, et al: Growth of pulmonary microvasculature in ventilated preterm infants. Am J Respir Crit Care Med 173: 204-211, 2006.

30. Maniscalco WM, Watkins RH, Pryhuber GS, Bhatt A, Shea C and Huyck H: Angiogenic factors and alveolar vasculature: development and alterations by injury in very premature baboons. Am J Physiol Lung Cell Mol Physiol 282: L811-L823, 2002.

31. Acarregui MJ, Penisten ST, Goss KL, Ramirez K and Snyder JM: Vascular endothelial growth factor gene expression in human fetal lung in vitro. Am J Respir Cell Mol Biol 20: 14-23, 1999.

32. Jakkula M, Le Cras TD, Gebb S, et al: Inhibition of angiogenesis decreases alveolarization in the developing rat lung. Am J Physiol Lung Cell Mol Physiol 279: L600-L607, 2000.

33. Hosford GE and Olson DM: Effects of hyperoxia on VEGF, its receptors and HIF-2alpha in the newborn rat lung. Am J Physiol Lung Cell Mol Physiol 285: L161-L168, 2003.

34. Le Cras TD, Markham NE, Tuder RM, Voelkel NF and Abman SH: Treatment of newborn rats with a VEGF receptor inhibitor causes pulmonary hypertension and abnormal lung structure. Am J Physiol Lung Cell Mol Physiol 283: L555-L562, 2002. 